Auror: Eventos reprodutivos pregressos como indicadores

Demétrio Antônio Gonçalves

DA SIIVA GOMES

ORIENTADOR:

Prof. Dr. Adriano Bueno Tavares

Resumo de tese

de risco para o desenvolvimento de diabetes mellitus

tipo-Il entre mulheres idosas

\author{
Past reproductive events as risk markers for the development \\ of diabetes mellitus type-II among elderly women
}

Palavras-chave

Resistência insulínica Diabetes mellitus tipo-ll

Macrossomia

Abortamento

Idosas

Keywords

Insulin resistance

Diabetes mellitus type $\|$

Macrosomy

Abortion

Elderly women

Dissertação de Mestrado apresentada ao Programa de Pós-Graduação Stricto Sensu em Gerontologia da Universidade Católica de Brasília - UCB, em 14 de dezembro de 2006.

A resistência insulínica é o fator etiopatogênico principal do diabetes mellitus tipo-II (DM-II), possuindo também papel central no desenvolvimento de distúrbios reprodutivos, como a síndrome dos ovários policísticos e o diabetes mellitus Gestacional, podendo estes últimos constituírem eventos-sentinela para o futuro aparecimento de DM-II.

OBJETIVO: identificar eventos reprodutivos pregressos como indicadores de risco para o desenvolvimento do DM-II em idosas. MÉTODOS: duzentas mulheres com idade $\geq 60$ anos foram selecionadas, tendo sido divididas em dois grupos: DM, compreendendo 75 idosas diabéticas (37,5\%), e, NDM, 125 idosas não-diabéticas (62,5\%). As participantes foram, então, avaliadas clinicamente, quando se levantou a história obstétrica pregressa para paridade, abortamento, abortamento freqüente (três ou mais eventos) e ocorrência de macrossomia fetal. RESULTADOS: observou-se que a paridade média foi de 3,2 partos para DM e 2,9 partos, em NDM ( $p=0,25$ ), com 4,0\% de nulíparas no grupo DM, e, $9,6 \%$, no grupo NDM (p=0,13). Quanto aos abortamentos, evidenciou-se maior freqüência deles no grupo DM, com $61,4 \%$, comparados aos 32,8\%, no NDM (p=0,001). Não houve diferença na ocorrência de abortamento freqüente, com 2,7\% em DM, e 0,8\% em NDM (p=0,30). Com relação à ocorrência de macrossomia fetal, observou-se que $49,3 \%$ das idosas diabéticas relataram esse evento pregresso, enquanto apenas $24,0 \%$ das não-diabéticas o fizeram ( $p=0,001$ ). CONCLUSÕES: a macrossomia fetal e a ocorrência de abortamento devem ser consideradas como eventos reprodutivos pregressos importantes na avaliação de ocorrência de DM-II entre mulheres idosas.

AUTOR:

Elvira Maria Mafaldo SoARes

ORIENTADORA:

Profa. Dra. Técia Maria de Olivera Maranhã

CO-ORIENTADOR

Prof. Dr. George Dantas de Azevedo

Resumo de tese

\title{
Avaliação da prevalência de síndrome metabólica, microalbuminúria e risco cardiovascular em mulheres com síndrome dos ovários policísticos
}

\author{
Prevalence of metabolic syndrome, microalbuminuria and cardiovascular \\ risk in women with polycystic ovary syndrome
}

Palavras-chave

Síndrome dos ovários policísticos Síndrome metabólica

Doença cardiovascular Microalbuminúria
Tese apresentada ao Programa de Pós-graduação em Ciências da Saúde da Universidade Federal do Rio Grande do Norte, para obtenção do tífulo de Doutor, em 17 de maio de 2007.

OBJETIVO: avaliar risco cardiovascular em mulheres com síndrome dos ovários policísticos (SOP), por meio da determinação da prevalência de síndrome metabólica (SM) e da pesquisa de microalbuminúria como marcador de possível dano renal precoce. MÉTODOS: estudo transversal envolvendo 102 mulheres (idade entre 20 e 34 anos) com diagnóstico de SOP de acordo com o Consenso Rotterdam. Para diagnóstico de SM foram adotados critérios do National Cholesterol Education Program's Adult Treatment Panel III (NCEP-ATP III). Para avaliação da microalbuminúria foi utilizada a relação albumina/creatinina (A/C), a partir dos níveis de albumina e creatinina em amostra isolada de urina. Análise estatística: teste $t$ de Student, $\chi^{2}$ e análises de correlação, adotando-se nível de significância 5\%. RESULTADOS: a prevalência de SM foi de 28,4\%, estando associada ao aumento do índice de massa corporal (IMC). Quanto aos componentes individuais da SM, evidenciou-se: HDL-colesterol $<50 \mathrm{mg} / \mathrm{dL} \mathrm{em} 69,6 \%$, circunferência da cintura $\geq 88 \mathrm{~cm}$ em $57,9 \%$, triglicerídeos $\geq 50 \mathrm{mg} / \mathrm{dL} \mathrm{em} 31,7 \%$, pressão arterial $\geq 130 / 85 \mathrm{mmHg}$ em 18,6\% e glicemia de jejum $\geq 110 \mathrm{mg} / \mathrm{dL} \mathrm{em} \mathrm{2,9 \%}$. Pelos limites convencionais para a relação A/C (3,5-35 mg/mmol), a microalbuminúria esteve presente em três pacientes $(3,3 \%)$. Entretanto, considerando diferentes pontos de corte estabelecidos em recentes estudos que demonstraram aumento do risco cardiovascular associado a níveis muito baixos da relação A/C, a prevalência em mulheres com SOP foi alta, variando de 17,7 a 43,3\% (A/C $\geq 0,58$ e $\geq 0,37 \mathrm{mg} / \mathrm{mmol}$, respectivamente). Mulheres com intolerância à glicose apresentaram relação A/C significativamente mais elevada, quando comparadas às mulheres normoglicêmicas. CONCLUSÕES: os dados evidenciam alta prevalência da SM e seus componentes em mulheres brasileiras com SOP. Adicionalmente, observou-se elevado percentual de mulheres com níveis de excreção urinária de albumina em faixas significativamente associadas com aumento do risco para eventos cardiovasculares. Em conjunto, esses dados alertam para a necessidade da abordagem interdisciplinar e multidisciplinar das pacientes com SOP, visando à instituição de medidas voltadas para a prevenção primária cardiovascular. 